\title{
Memory Impairment Due to Stroke
}

\author{
Shinichiro Maeshima ${ }^{1}$ Aiko Osawa² \\ ${ }^{1}$ Kinjo University, Ishikawa, Japan; ${ }^{2}$ Department of Rehabilitation Medicine, \\ National Center for Geriatrics and Gerontology, Aichi, Japan
}

Author for correspondence: Shinichiro Maeshima, Kinjo University, Ishikawa, Japan. Email: shinichiromaeshima@gmail.com

Doi: https://doi.org/10.36255/exonpublications.stroke.memoryimpairment.2021

\begin{abstract}
Stroke impairs episodic memory, while retaining immediate and remote memory. Cerebral hemorrhage/infarction in the Papez and Yakovlev circuits (episodic memory) manifests as memory loss. Extensive medial temporal lobe damage impairs retrieval of old memories. Hippocampal damage causes anterograde amnesia. Damage to the hippocampus and parahippocampal gyri causes severe retrograde amnesia. Retrosplenial lesions may cause memory loss if the fibrous communication between the hippocampus and anterior thalamic nucleus is impaired. Basal forebrain amnesia impairs recall but the aspect providing retrieval clues remains relatively intact. Corpus striatum and basal forebrain damage results in serious memory loss. Other cognitive functions require evaluation using intelligence (WAIS-IV) and executive function tests, along with memory loss assessments. The WMS-R can evaluate memory after stroke. The RBMT elucidates the nature of memory loss, especially in the elderly. Cognitive rehabilitation with repetitive training and internal-memory strategies aims to activate the memory processes. External strategies and environmental adjustments provide effective clues and replicate the environment. Rehabilitation protocols should directly solve problems encountered in daily life. This chapter provides an overview of the anatomical basis of memory, pathophysiology of underlying memory loss, and assessment for stroke.
\end{abstract}

Keywords: amnesia; cerebral infarction; cerebral hemorrhage; memory impairment; stroke

In: Stroke. Dehkharghani S (Editor). Exon Publications, Brisbane, Australia. ISBN: 978-0-6450017-6-1; Doi: https://doi.org/10.36255/exonpublications.stroke.2021

Copyright: The Authors.

License: This open access article is licenced under Creative Commons Attribution-NonCommercial 4.0 International (CC BY-NC 4.0) https://creativecommons.org/licenses/by-nc/4.0/ 


\section{INTRODUCTION}

Memory is one of the most complex functions of the human brain that entails storage of information acquired from learning or experience, enabling the use of that information in the future (1). Memory is divided into a three-stage process comprised of memorizing (encoding), retention (storage) and regeneration (recall). Various problems associated with memory, including forgetting tasks, misplacing items, and failure to keep engagements make daily life more difficult for individuals with higher brain dysfunction and dementia. Cerebral infarction and brain hemorrhage are the most common causes of memory loss. However, memory loss can also be caused by subarachnoid hemorrhage, brain injury, and degenerative diseases. This chapter explains the pathophysiology of memory loss and the necessary assessments for stroke in clinical practice.

\section{CLASSIFICATION OF MEMORY}

Memory organically links an individual's activities over time. The classification of the development of memory over time is shown in Figure 1. The clinical terms used to describe memory include immediate memory, recent memory, and remote memory. Immediate memory is the memory of an experience that is stored temporarily without deep processing, without any interference with recall. The retained information is always at the surface of the conscious mind. This memory is specifically assessed using forward/backward digit span tasks (repeating a number series) and immediate recall of a series of spatial locations. Recent memory is memory that is retained for a longer period than immediate memory, but the time interval

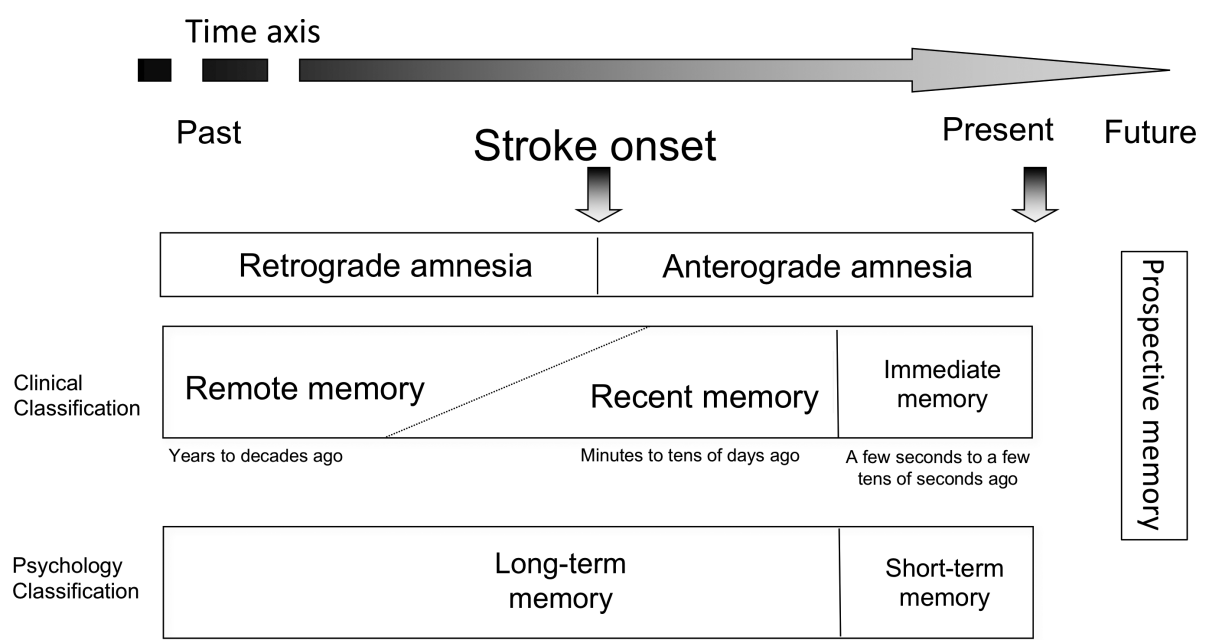

Figure 1. Classification of the development of memory over time. The classification of the development of memory over time is shown. The clinical terms used to describe memory include immediate memory, recent memory, and remote memory. 
between encoding and recall is not clearly defined. Other cognitive tasks interfere with retrieval of memory because the information must be recalled after a period of time following memorizing (ranging from a few minutes to a few days). Therefore, the retained information disappears from consciousness once. In clinical practice, recent memory is assessed by enquiring about the content of the patient's evening meal on the previous day. Remote memory is memory retained for an even longer duration than that of recent memory. Although there is no clear definition, remote memory is assessed by asking an individual about their life history.

Short-term memory and long-term memory are terms used in the field of psychology (2). Temporally, short-term memory is stored temporarily, exists for a few seconds to a few minutes, and eventually disappears. Thus, this term has virtually the same meaning as immediate memory from a clinical and neurological perspective. Long-term memory is converted from short-term memory and stored almost indefinitely. Long-term memory includes both recent memory and remote memory. Long-term memory that relates to information that exists on the surface of the conscious mind is called explicit memory, while memory related to information that exists deep within the conscious mind is called implicit memory (3). Explicit memory is formed via the sensory organs and is also known as conceptual memory. Explicit memory represents declarative memories that can be easily articulated (4). Declarative memory can be further classified into semantic memory and episodic memory (5). Semantic memory is related to general knowledge of various facts and events and is referred to as conceptual memory. On the other hand, episodic memory refers to a series of memories associated with the passage of time and is related to events experienced by the individual (Figure 2). Implicit memory, which is also called nondeclarative memory, includes procedural memory, priming, and the conditioned reflex. However, these memories are difficult to articulate and elucidate. Procedural memory includes memories related to physical motor functions, such as riding a bicycle and playing a musical instrument.

\section{Memory impairment (amnesia)}

In stroke medicine, memory impairment generally refers to episodic memory impairment rather than the impairment of semantic or procedural memory. Stroke patients with memory impairment experience loss of recent memory but retain

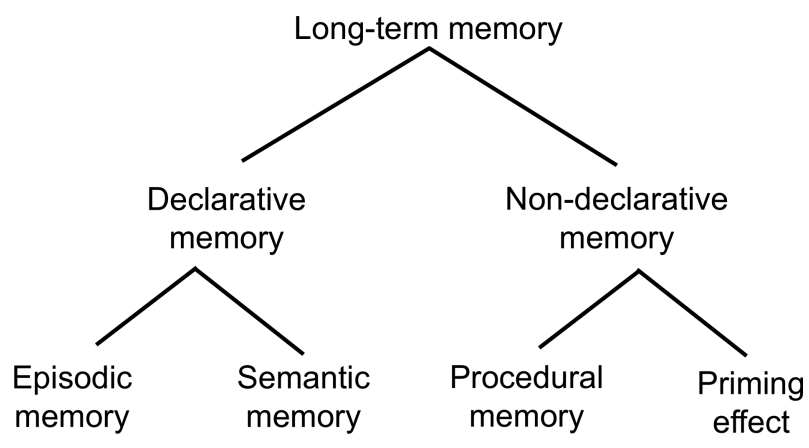

Figure 2. Classification by memory content. 
immediate memory. Their memory of the distant past, known as remote memory, also remains relatively intact. Memory loss in stroke patients can also be associated with disorientation due to anterograde amnesia, stemming from the inability to acquire or accumulate new memories after the episode of stroke, and retrograde amnesia where the patient is unable to recall memory acquired before the stroke, as well as confabulation, attention disorder, and changes in personality.

\section{ANATOMICAL BASIS OF MEMORY AND LESIONS CAUSING MEMORY IMPAIRMENT}

The neural circuits related to episodic memory include the Papez circuit (medial limbic circuit) centred around the hippocampus, anterior thalamic nucleus, medial mammillary nucleus, and parahippocampal gyri, as well as the Yakovlev circuit (ventrolateral limbic circuit), centred on the amygdala, medial thalamic nucleus and orbitofrontal cortex. Although each circuit is independent of the other (6), they are very closely related (Figure 3), and cerebral haemorrhage or cerebral infarction at these sites manifests as memory loss (Figure 4).

The posterior cerebral artery is formed by the confluence of the left and right bifurcation of the rostral end of the basilar artery, which runs laterally, superior to the cerebral peduncle, joins the posterior communicating artery, and subsequently detours along the lateral surface of the midbrain. It traverses the upper surface of the cerebellar tentorium and extends to the medial and inferior surfaces of the

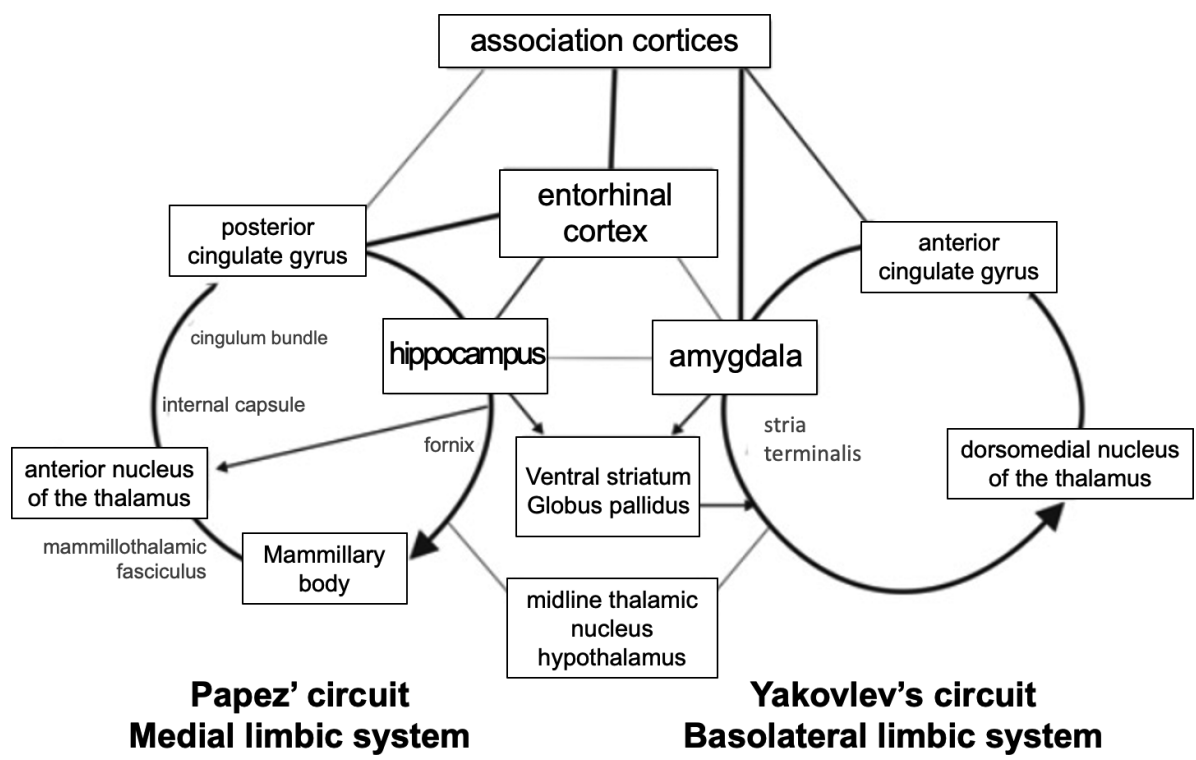

Figure 3. Schematic diagram of Papez' circuit and Yakovlev's circuit. The neural circuits related to episodic memory include the Papez circuit (medial limbic circuit) and the Yakovlev circuit (ventrolateral limbic circuit). Although each circuit is independent of the other, they are very closely related. 


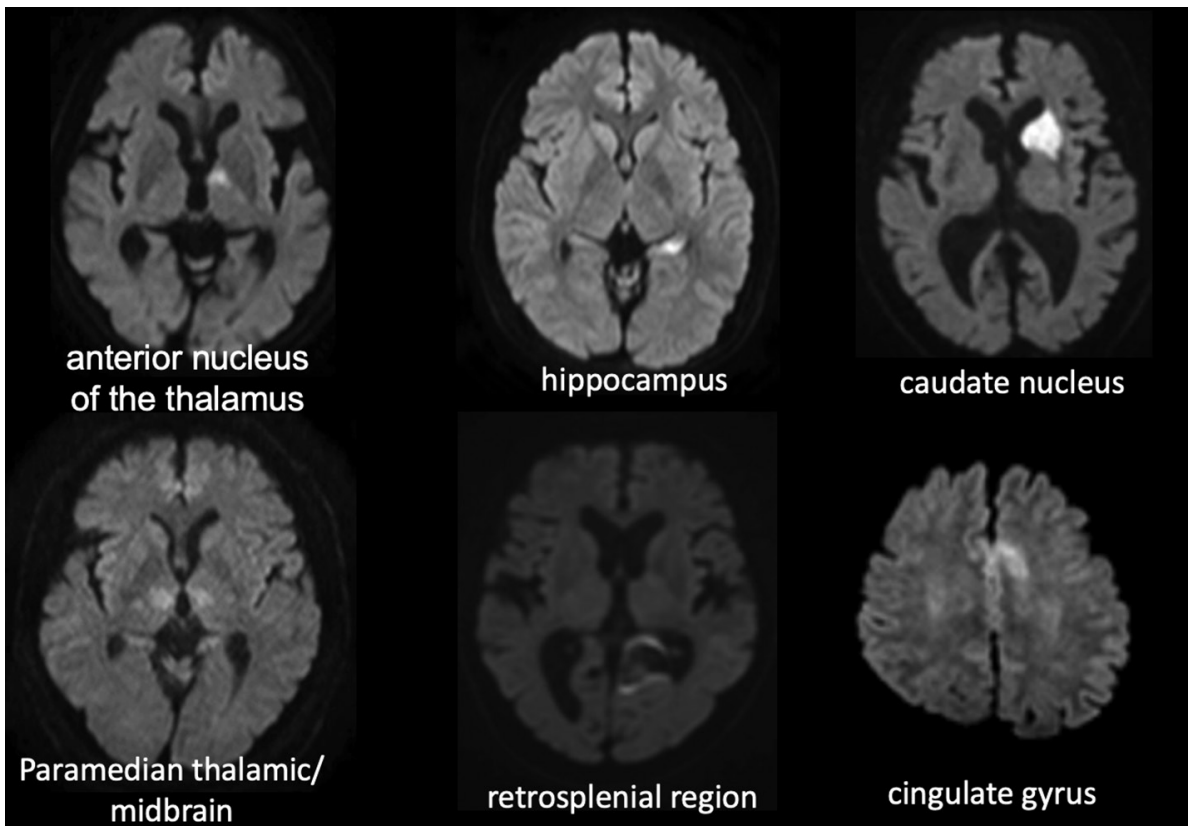

Figure 4. Lesions in patients with episodic memory impairment. Memory impairment occurs when strokes occur in areas associated with episodic memory, including the hippocampus, anterior thalamic nucleus, medial papillary nucleus, parahyppocampal gyrus, amygdala, medial thalamic nucleus, and orbitofrontal cortex.

temporal and occipital lobes. The lateral and medial occipital arteries originate from the posterior cerebral artery, and perfuse the hippocampus, parahippocampal gyri, fornix, and posterior region of the cingulate gyri.

The manifestation of memory loss associated with medial temporal lobe lesions is variable. The hippocampus does not store or retrieve memory; instead, it plays an important role in encoding memory and is involved in consolidating memories formed within the recent past (7). Damage localized to the hippocampus presents as anterograde amnesia, but is not always associated with retrograde amnesia, and old memories are often retained (8). Widespread damage to the medial aspect of the temporal lobe, such as the hippocampus and parahippocampal gyrus, impairs retrieval of old memories and results in severe backward amnesia (9).

The posterior cingulate gyrus has fibrous connections with the anterior cingulate gyrus, medial aspect of the temporal lobe, prefrontal cortex, posterior parietal lobe, thalamus, and caudate nucleus. Even lesions in the retrosplenial region present with memory loss, known as retrosplenial amnesia, which is thought to be caused by impairment of the fibrous communication of the memory circuit between the hippocampus and the anterior nucleus of thalamus due to damage to the posterior cingulate gyrus (10). It reportedly occurs more frequently as a sequela of hemorrhage than that of infarction $(11,12)$.

The thalamus receives vascular supply from the anterior and posterior circulation (13). The thalamotuberal arteries (polar artery) that branch from the posterior communicating artery perfuse the anterior nucleus of thalamus, and damage to this area presents as episodic memory loss. The paramedian thalamic artery, 
which originates from the posterior cerebral artery, traverses the dorsomedial nucleus of thalamus, and may also branch bilaterally from the posterior cerebral artery on one side (14). Lesions of the bilateral dorsomedial nucleus of the thalamus can result in severe impairment of consciousness. Damage to these areas of the thalamus due to cerebral infarction, surgical invasion (15), and/or cerebral hemorrhage (16) causes memory loss (Figure 5).

On the other hand, memory loss due to subarachnoid hemorrhage involves a myriad of factors, which include associated intracerebral hematoma, cerebral vasospasm, and hydrocephalus. Studies have furthermore reported postoperative amnesia in more than half of patients who underwent surgery $(17,18)$; generally, the more severe the symptoms, the worse the functional prognosis. Moreover, such patients are also prone to symptoms such as memory loss. Subarachnoid hemorrhage due to rupture of a cerebral aneurysm in the anterior cerebral artery system or vertebral artery system with associated parenchymal injury are more likely to cause memory loss than rupture of the internal carotid artery system or middle cerebral artery system. Basal forebrain amnesia after rupture of an anterior communicating artery aneurysm is the most commonly observed clinical sequela (19).

The basal forebrain consists of the lateral and medial septal nuclei, diagonal band of Broca, and the nucleus basalis of Meynert, and is thought to contain cholinergic neurons that project into the cerebral cortex, hippocampus, and amygdala and regulate the regional cerebral blood flow. This region is perfused by the perforating branches of the anterior cerebral artery or anterior communicating artery. The anterior communicating artery (20) has an average of 5.4 perforating branches, which are divided into the subcallosal, hypothalamic, and chiasmatic branches (21). The subcallosal branch, which perfuses the bilateral subcallosal areas, is associated with memory loss. Memory loss can be caused by damage to these perforating branches during surgical procedures for subarachnoid hemorrhage caused by rupture of an anterior communicating artery aneurysm (22), or vascular spasm. The basal forebrain is involved in the regeneration of memory content (23); thus, basal forebrain amnesia impairs only the recall process (24), leaving the part of recall that provides retrieval clues relatively intact (25). Concomitant damage to the corpus striatum and basal forebrain is known to

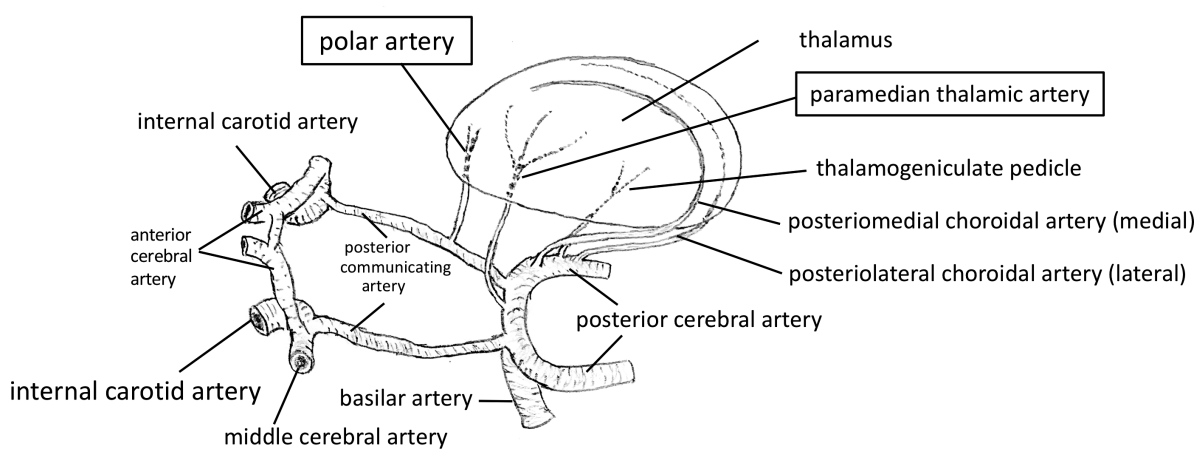

Figure 5. Blood supply to the thalamus. The thalamus receives blood supply from the anterior and posterior circulation. Inadequate perfusion of the thalamus, cerebral infarction and hemorrhage can cause memory loss. 
cause more serious memory loss. Therefore, the recurrent artery of Heubner and the perforating branches of the middle cerebral artery, which nourish the caudate nucleus, also have important involvement in memory.

\section{ASSESSMENT OF MEMORY IMPAIRMENT}

The Wechsler Memory Scale-Revised (WMS-R) is a comprehensive memory test composed of five indices: (i) verbal memory, (ii) visual memory, (iii) general memory, (iv) attention/concentration, and (v) delayed recall. As with the Wechsler Adult Intelligence Scale Fourth Edition (WAIS-IV) intelligence test, the results are expressed using a score with an average of value of 100 . If the patient has aphasia, the final score will be significantly reduced for verbal memory, while unilateral spatial neglect or constructional apraxia will significantly reduce the score for the visual memory item. Therefore, it is necessary to investigate the presence of these conditions and disorders before subjecting the patient to the test.

The Rivermead Behavioural Memory Test (RBMT) does not simply assess the individual's capacity to register memory, but rather seeks to clarify the nature of memory loss by evaluating it in a manner that resembles memory required for daily life, such as people's first and last names, faces, everyday engagements, everyday tasks, and directions. The RBMT comprises four different types of tasks, and the test can be repeated in a short period of time. The RBMT is an indispensable instrument for assessing the memory of elderly individuals by envisioning their daily lives at home. It is recommended that other cognitive functions should also be evaluated using intelligence tests such as WAIS-IV along with executive function tests, in addition to assessing memory function in patients with memory loss.

\section{REHABILITATION}

Although cognitive rehabilitation may lessen the impact of memory loss after stroke on daily life immediately after treatment, there is a scarcity of clear evidence to support its effectiveness. However, the methodology and effect of memory loss rehabilitation are widely recognized (26). Repetitive training and internal memory strategies (visual image method, PQRST method) exist as "approaches aiming to activate the memory process". External strategies and environmental adjustments also exist, which are adopted as "approaches based on providing effective clues and the environment". The external strategy attempts to compensate for the memory process by using external aids including memoranda, notes, shopping lists and written messages. Environmental adjustment seeks to reduce memory-dependent behavior and eliminate difficulties encountered in daily life by correcting or modifying the patient's environment. Specific strategies include acquisition and errorless learning of area-specific knowledge limited to information required for daily life such as directions to hospitals and learning schedules, environmental adjustments such as labelling and setting up boxes containing valuables, and use of external aids such as memory notes, etc. Prospective memory is important in daily life to remember future tasks in a timely manner. Recently, however, mobile devices such 
as cell phones and smart phones have become smaller and smaller, and individuals are using them on a daily basis. Thus, training now involves using the alarm function and scheduling function on these devices. The American Congress of Rehabilitation Medicine recommends application-based external compensation for severe memory loss after stroke (27). Either way, treatment programs should be designed to ensure that rehabilitation of memory loss can directly solve problems encountered by patients in their daily lives.

\section{CONCLUSION}

Memory loss due to stroke is chiefly episodic in presentation. Areas of the brain related to memory are controlled by the anterior and posterior circulation, and ischemic or hemorrhagic lesions in these areas are responsible for memory loss. The methodology and efficacy of cognitive rehabilitation for memory loss after stroke are widely accepted, despite the lack of clear evidence that supports the efficacy of rehabilitation. Treatment programs should be designed to solve problems encountered by patients in their daily life.

Conflict of interest: The authors declare no potential conflicts of interest with respect to research, authorship, and/or publication of this chapter.

Copyright and Permission Statement: The authors confirm that the materials included in this chapter do not violate copyright laws. Where relevant, appropriate permissions have been obtained from the original copyright holder(s), and all original sources have been appropriately acknowledged or referenced.

\section{REFERENCES}

1. Squire LR, Zola-Morgan S. The medial temporal lobe memory system. Science. 1991;253(5026): 1380-6. https://doi.org/10.1126/science.1896849

2. Atkinson RC, Shiffrin RM. Human memory: A proposed system and its control processes. Psychology of learning and motivation. 1968;2(4):89-195. https://doi.org/10.1016/S0079-7421(08)60422-3

3. Tulving E, Schacter DL. Priming and human memory systems. Science. 1990;247(4940):301-6. https://doi.org/10.1126/science.2296719

4. Squire LR, Zola SM. Structure and function of declarative and nondeclarative memory systems. Proc Natl Acad Sci. 1996;93(24):13515-22. https://doi.org/10.1073/pnas.93.24.13515

5. Squire LR, Zola SM. Episodic memory, semantic memory, and amnesia. Hippocampus. 1998;8(3): 205-11. https://doi.org/10.1002/(SICI)1098-1063(1998)8:3<205::AID-HIPO3>3.0.CO;2-I

6. Livingston KE, Escobar A. Anatomical bias of the limbic system concept; a proposed reorientation. Arch Neurol. 1971;24:17-21. https://doi.org/10.1001/archneur.1971.00480310045003

7. Squire LR. Memory and the hippocampus: A synthesis from findings with rats, monkeys, and humans. Psychol Rev. 1992;99:195-231. https://doi.org/10.1037/0033-295X.99.2.195

8. Fujii T, Moscovitch M, Nadel L. Memory consolidation, retrograde amnesia, and the temporal lobe, In Handbook of Neuropsychology. Volume 2. 2nd Edition. Edited by Boller F and Grafman J: Amsterdam: Elsevier;2000. p. 223-50.

9. Bright P, Buckman J, Fradera A, Yashimasu H, Colchester AC, Kopelman MD: Retrograde amnesia in patients with hippocampal, medial temporal, temporal lobe, or frontal pathology. Learn Mem. 2006;13:545-57. https://doi.org/10.1101/lm.265906 
10. Valenstein E, Bowers D, Verfaellie M, Heilman KM. Retrosplenial amnesia. Brain 1987;110(6):1631-46. https://doi.org/10.1093/brain/110.6.1631

11. Maeshima S, Ozaki F, Masuo O, Yamaga H, Okita R, Moriwaki H. Memory impairment and spatial disorientation following a left retrosplenial lesion. J Clin Neurosci. 2001;8:450-1. https://doi. org/10.1054/jocn.2000.0801

12. Maeshima S, Osawa A, Yamane F, Yoshihara T, Kanazawa R, Ishihara S. Retrosplenial amnesia without topographic disorientation caused by a lesion in the nondominant hemisphere. J Stroke Cerebrovasc Dis. 2014;23(3):441-5. https://doi.org/10.1016/j.jstrokecerebrovasdis.2013.03.026

13. Bogousslavsky J, Caplan LR. Vertebrobasilar occlusive disease: review of selected aspects. Cerebrovasc Dis. 1993;3(4):193-205. https://doi.org/10.1159/000108701

14. Percheron G. Arteries of the human thalamus. II. Arteries and paramedian thalamic territory of the communicating basilar artery. Revue Neurologique. 1976;132(5):309-24.

15. Maeshima S, Ueyoshi A, Tsuura M, Takemoto H, Itakura T, Yoshida M, et al. Transient aphasia and persistent amnesia after surgery for internal carotid artery-posterior communicating artery aneurysm. J Clin Neurosci. 2002;9(6):710-3. https://doi.org/10.1054/jocn.2001.1065

16. Maeshima S, Ozaki F, Okita R, Yamaga H, Okada H, Kakishita K, et al. Transient crossed aphasia and persistent amnesia after right thalamic haemorrhage. Brain Injury. 2001;15(10):927-33. https://doi. org/10.1080/02699050110065646

17. Lindqvist $G$, Norlén $G$. Korsakoff's syndrome after operation on ruptured aneurysm of the anterior communicating artery. Acta Psychiatr Scand. 1966;42:24-34. https://doi.org/10.1111/j.1600-0447.1966. tb01913.x

18. Ljungreen B, Sonesson B, Säveland H, Brandt L. Cognitive impairment and adjustment in patients without neurological deficits after aneurismal SAH and early operation. J Neurosurg. 1985;62:673-9. https://doi.org/10.3171/jns.1985.62.5.0673

19. Norlen G, Olivecrona H. Surgical treatment of aneurysms of the anterior communicating artery. J Neurosurg. 1953;10:634-50. https://doi.org/10.3171/jns.1953.10.6.0634

20. Crowell RM, Morawetz RB. The anterior communicating artery has significant branches. Stroke. 8(2):272-3,1977. https://doi.org/10.1161/01.STR.8.2.272

21. Serizawa T, Saeki N, Yamaura A. Microsurgical anatomy and clinical significance of the anterior communicating artery and its perforating branches. Neurosurgery. 1997;40(6):1211-8. https://doi. org/10.1097/00006123-199706000-00019

22. Gade A. Amnesia after operations on aneurysms of the anterior communicating artery. Surg Neurol 1982;18(1):46-9. https://doi.org/10.1016/0090-3019(82)90013-1

23. Fujii T, Okuda J, Tsukiura T, Ohtake H, Miura R, Fukatsu R, Suzuki K, Kawashima R, Itoh M, Fukuda H, Yamadori A. The role of the basal forebrain in episodic memory retrieval: a positron emission tomography study. Neuroimage. 2002;15(3):501-8. https://doi.org/10.1006/nimg.2001.0995

24. Moscovitch M, Melo B. Strategic retrieval and the frontal lobes: Evidence from confabulation and amnesia. Neuropsychologia. 1997;35(7):1017-34. https://doi.org/10.1016/S0028-3932(97)00028-6

25. Damasio AR, Graff-Radford NR, Eslinger PJ, Damasio H, Kassell N. Amnesia following basal forebrain lesions. Arch Neurol. 1985;42(3):263-71. https://doi.org/10.1001/archneur.1985.04060030081013

26. Cicerone KD, Goldin Y, Ganci K, Rosenbaum A, Wethe JV, Langenbahn DM, et al. Evidence-Based Cognitive Rehabilitation: updated review of the literature from 2009 through 2014. Arch Phys Med Rehabil 2019;100:1515-33. https://doi.org/10.1016/j.apmr.2019.02.011

27. das Nair R, Martin KJ, Lincoln NB. Cognitive rehabilitation for memory deficits after stroke. Cochrane Database Syst Rev. 2016 Sep 1;9(9):CD002293. doi: 10.1002/14651858.CD002293.pub3. https:// doi.org/10.1002/14651858.CD002293.pub3 
